\title{
THE EFFECTS OF MECHANICAL VIBRATION ON CARDIAC MORPHOGENESIS IN THE STAGE 14 CHICK EMBRYO
}

\author{
Shizen Ishikawa, M.D., Yoshinari Inaba, M.D., Steve Kargas, M.S* \\ Hiroshi Chikaoka, M.D., and Dong S. Park, M.D.
}

\begin{abstract}
The primitive cardiac loop of the stage 14 (Hamburger and Hamilton) chick embryo was subjected to brief vibratory stimuli that resulted in a broad spectrum of cardiovascular malformations. During the 17 th day of incubation, both survival and anomaly rates were evaluated. Compared with control embryos $(92 \%)$, vibration elicited mortality $(75 \%)$ that was statistically highly significant $(\mathrm{p}<0.001)$. In addition, the anomaly rate $(94 \%)$ among surviving embryos was also highly significant $(p<0.001)$ relative to controls. Defects included straddling AV valves, variable sizes of VSD and DORV. Several possible explanations are presented concerning the etiology of these vibrationinduced malformations.
\end{abstract}

$T^{n}$ HE study of cardiac morphogenesis has been excellently reviewed by several authors., The pathogenesis of cardiovascular malformations in the chick embryo has also been studied, especially with regard to hemodynamic alterations $3^{3-5}$ These investigations have provided valuable insight into the understanding of this complex morphogenetic process. However, more basic research concerning the teratogenic mechanism remains to be correlated with normal cardiogenesis.

To investigate the mechanical effect of vibration on cardiovascular development and the subsequent induction of malformations, we utilized the chick embryo as an experimental model. Gessner ${ }^{6}$ has previously established that temporary mechanical displacement of the contruncus in the stage 19-20 chick embryo produced vary-

\footnotetext{
Key Words:

Vibration

Chick embryo

Cardiac morphogenesis

Heart

Embryology
}

ing degrees of aortic dextroposition. We have expanded on this concept by applying the vibratory stimulus to the primitive cardiac loop at an earlier embryonic stage (stage 14) and thereby produced a variety of malformations. In order to stimulate the primitive heart, we devised a mechanical vibrator which interferes with normal embryological development.

\section{MATERIALS AND METHODS}

Fertile White Leghorn chicken eggs (50-60 $\mathrm{gm} / \mathrm{egg}$ ) were obtained from a local hatchery and incubated under normal temperature $\left(37.5^{\circ} \mathrm{C}\right)$ and relative humidity conditions $(60 \%)$ in a forced air-electric cabinet. After 50-56 hours of incubation (Hamburger and Hamilton stage 14), a small hole was carefully made in the pointed end of the egg using sharp forceps. Two $\mathrm{ml}$ of albumin was then withdrawn with a sterile syringe to permit the embryo to fall away from the shell membrane. A $1-\mathrm{cm}^{2}$ piece of the egg shell and membrane was removed with forceps, which exposed the embryo and interior of the

(Received December 27, 1985; accepted March 31, 1986)

Department of Pediatrics, Showa University, Tokyo, Japan; ${ }^{*}$ Department of Pathology, University of Wisconsin Medical School, Madison, WI., U.S.A.

Mailing address: Shizen Ishikawa, M.D., Department of Pediatrics, Showa University, 1-5-8, Hatanodai, Shinagawa-ku, Tokyo 142, Japan 


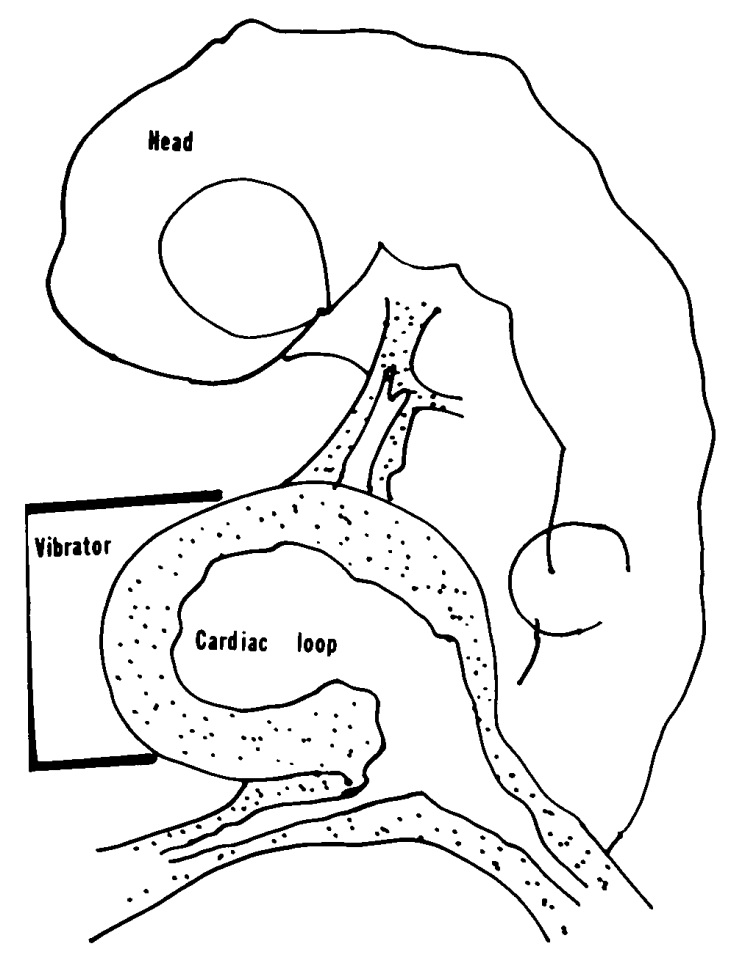

Fig.1. The primitive cardiac loop is shown with the vibrator placed in a vertical position. egg. Subsequently, a part of the chorioallantoic membrane of the embryo was microdissected away from the surface of the egg. Any deformed, hemorrhagic, or abnormally positioned embryos were eliminated from the study. Afterwards, the eggs were sealed by cellophane tape to prevent exogenous bacterial infection and dehydration, and at the same time to provide rapid access to the embryo.

The mechanical vibrator used in our study consisted of two pieces of copper wire separated by $1.5 \mathrm{~mm}$. After these tips were sterilized with alcohol, they were introduced through the observation window and accurately positioned on the embryo using a dissecting microscope. Our method utilized the direct application of vibratory stimuli between two points along the conotruncus and apex of the cardiac loop (Fig. 1). The degree of vibration applied to the primitive cardiac loop was $2 \mathrm{~mm}$ in amplitude at a frequency of 200 hertz. We elected to apply vibratory stimuli for 90 seconds to each egg since it yielded the highest survival rate concurrently with the highest malformation rate. Following



B

Vibrator

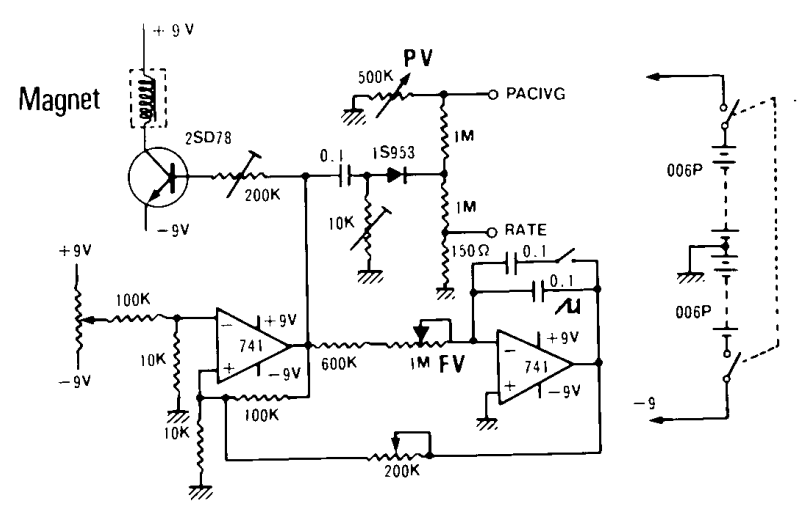

Fig.2. A) The device used to apply vibration to the embryonic chick heart.

B) Detailed electronic schematic diagram of the mechanical vibrator. Frequency Volume (FV); Pacing Volume (PV)

Japanese Circulation Journal Vol. 50, October 1986 


\section{Vibration 165 cases}

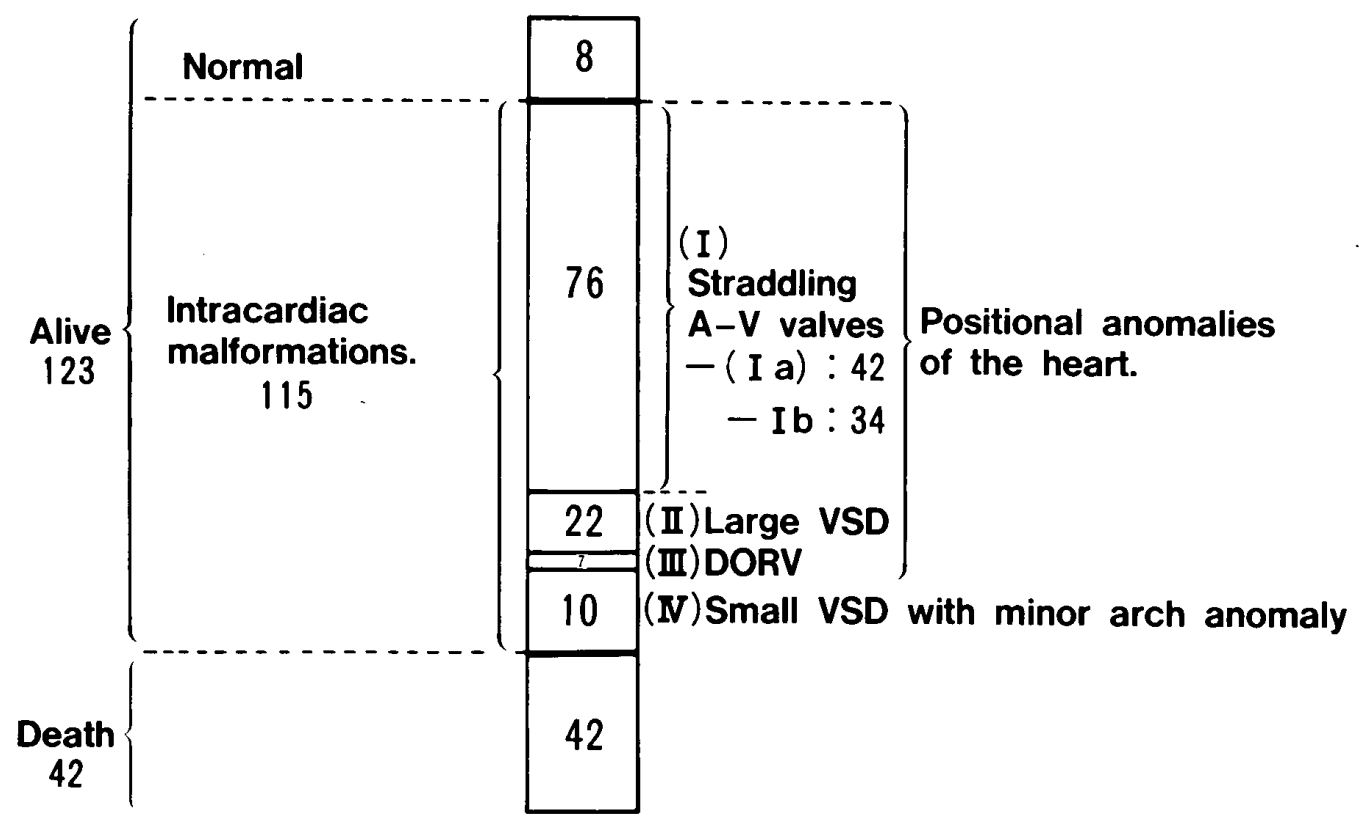

Fig.3. A summary of our data on the various intracardiac malformations associated with positional anomalies induced in our experiments. Roman numerals indicate the total number of eggs that were treated with vibration. Arabic numerals (I-IV) represent the major Groups used to classify the malformations.

vibration, the eggs were reincubated and left undisturbed until day 17 of incubation, at which time the survivors were sacrificed and examined for cardiac plus aortic arch malformations.

The device used to apply mechanical vibration to the cardiac loop was constructed using a variable frequency generator, an electromagnet, and a digital frequency meter (Fig. 2). The electronic oscillator was power-driven by a battery. This oscillator produced an alternating square wave which repeatedly turned the electromagnet either on or off, thereby causing the tips to vibrate back and forth. In order to control vibrating amplitude, an adjusting knob was used to change the position of the electromagnet.

A total of 165 embryos were employed in the vibration experimental group and another 100 embryos were used as controls. In the control group, the microelectrode tips were inserted for 90 seconds along the primitive cardiac loop without applying vibration, and then the eggs were reincubated until day 17 . It should be emphasized that the reason we experimented with eggs at stage 14 of embryonic development was to administer the vibrating stimuli concomitantly with cardiac myofibrillar morphogenesis. Since our control anomaly rate (eggs being windowed but not exposed to vibration) was relatively low, we felt that early windowing technique fell within statistically acceptable limits.

Scanning electron microscopy (SEM) was used to demonstate abnormal cardiac morphogenesis after vibration treatment. Hearts from surviving embryos were injected with $2.5 \%$ phosphatebuffered glutaraldehyde to wash out the blood and to preserve the internal morphology. Embryos were then fixed in $10 \%$ formalin and sectioned for SEM.

We employed the chi-square $2 \times 2$ contingency test to statistically analyze the data. A p-value of less than 0.05 was regarded as an indication that a statistical difference exists between values.

\section{RESULTS}

A comparison between the embryonic survival rates in the experimental group that received vibration in contrast to the controls was statistically highly significant $(\mathrm{p}<0.001)$ : $75 \%(123 /$ $165)$ and $92 \%(92 / 100)$, respectively. Figure 3 illustrates the findings of 165 treated embryos. Among the surviving 123 embryos examined, 115 cases (94\%) exhibited abnormal morphology 


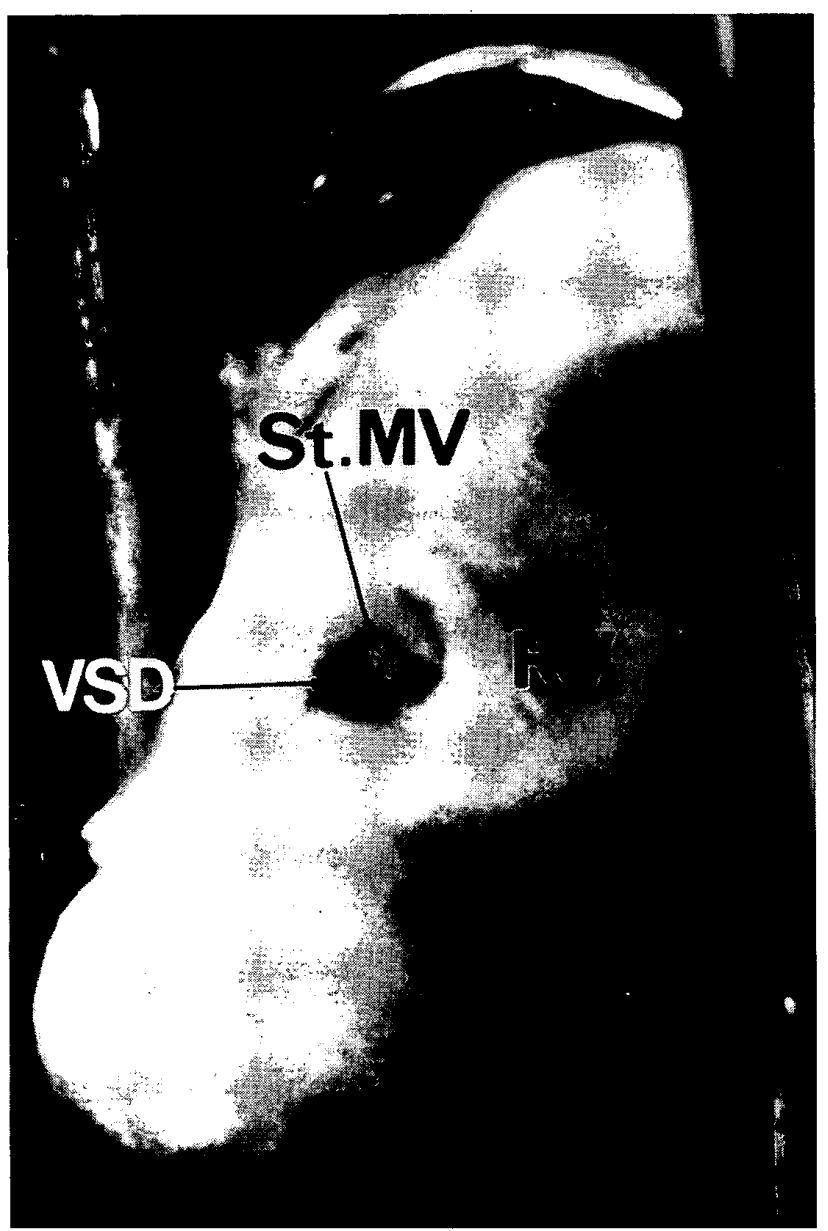

Fig.4. A mitral valve straddling (St. MV) through a Ventricular Septal Defect (VSD) into a hypoplastic right ventricle (RV). of the heart or great vessels. In the control group, $5 / 92(5 \%)$ surviving embryos had a ventricular septal defect which was located at the uppermost lateral border of the crista supraventricularis. This location was a common site of spontaneous VSD in the chick embryo. The high incidence of large VSD in the experimental group compared with that of the control group can be appreciated. There was also a highly significant difference $(p<0.001)$ between the vibration-induced anomaly rate and the control embryo anomaly rate. The surviving embryos in the experimental group were classified into four major wellrecognizable groups (I-IV). In order to classify adequately hearts with straddling valves, either mitral or tricuspid, frequently occurring in our experiment, and other minor anomalies without straddling, it is convenient to distinguish these malformations into four basic categories. Positional anomalies of the heart were placed in groups I-III.

In Group I, the main cardiac malformation among the surviving embryos involved straddling AV valves $(76 / 123=62 \%)$. We subdivided this group into two categories: Group Ia embryos $(42 / 76=55 \%)$ exhibited mitral valvular elements (the annulus, leaflet, and chordae tendinae) that partially straddled a moderately hypoplastic right ventricular chamber (Fig. 4). In addition, the left ventricular inflow and outflow tracts became

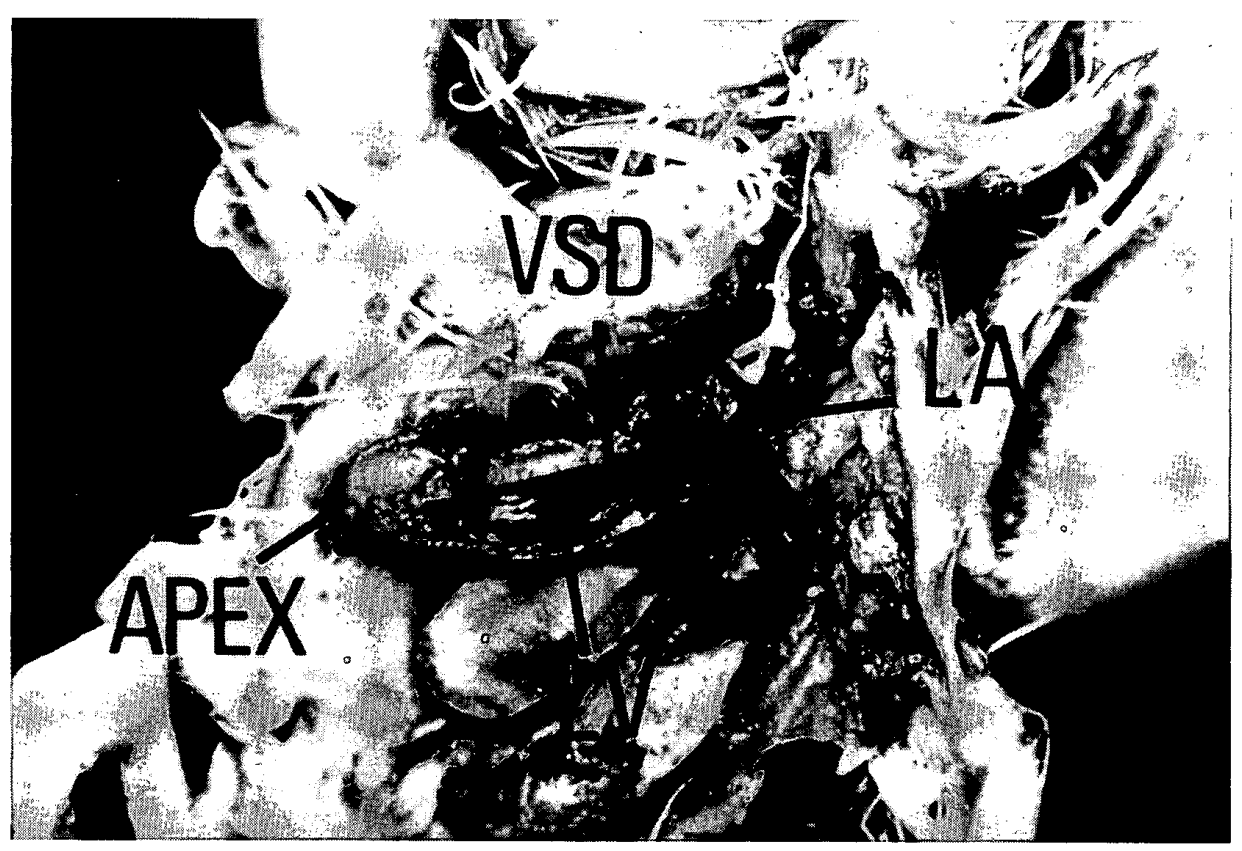

Fig.5. The apex of the heart is totally displaced toward the right chest. The visceral organs are located normally. Abbreviations: $\mathrm{VSD}=$ ventricular septal defect, $\mathrm{LA}=\mathrm{left}$ atrium, LV = left ventricle 


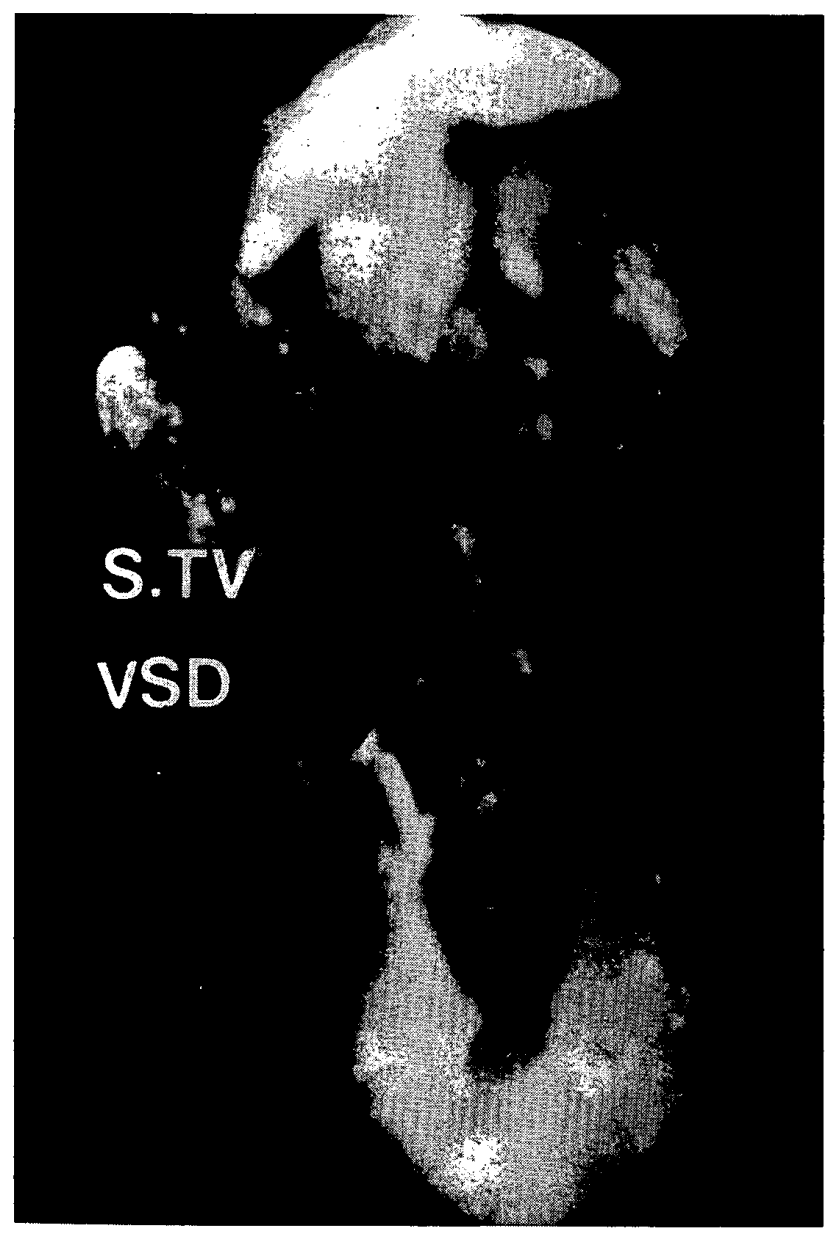

Fig.6. A tricuspid valve partially straddling (S. TV) through a large Ventricular Septal Defect (VSD) into a hypoplastic left ventricle. Both the right ventricular inflow and outflow tracts are elongated.

elongated. Of these Group Ia embryos, isolated dextrocardia was noted in $78 \%$ (33/42) (Fig. 5). The remaining nine cases showed mesocardia with an undetermined apex. Group $\mathrm{Ib}$ embryos $(34 / 76=45 \%)$ may be described as having a tricuspid valve orifice being displaced through a large ventricular septal defect that straddled into a relatively hypoplastic left ventricular chamber (Fig. 6). Both the right ventricular inflow and outflow tracts also became elongated, and isolated dextrocardia was noted in $74 \%(25 / 34)$. The remaining nine cases in this subdivision presented with mesocardia. A ventricular septal defect was an essential lesion of straddling tricuspid or mitral valves. Two different types of VSD were frequently recognized, one with involvement of the inlet septum (Group Ib) and another without involvement of the inlet septum (Group Ia).

In Group II, a large isolated ventricular septal defect (VSD) was located below the crista supraventricularis in 22 cases $(22 / 123=18 \%)$. No

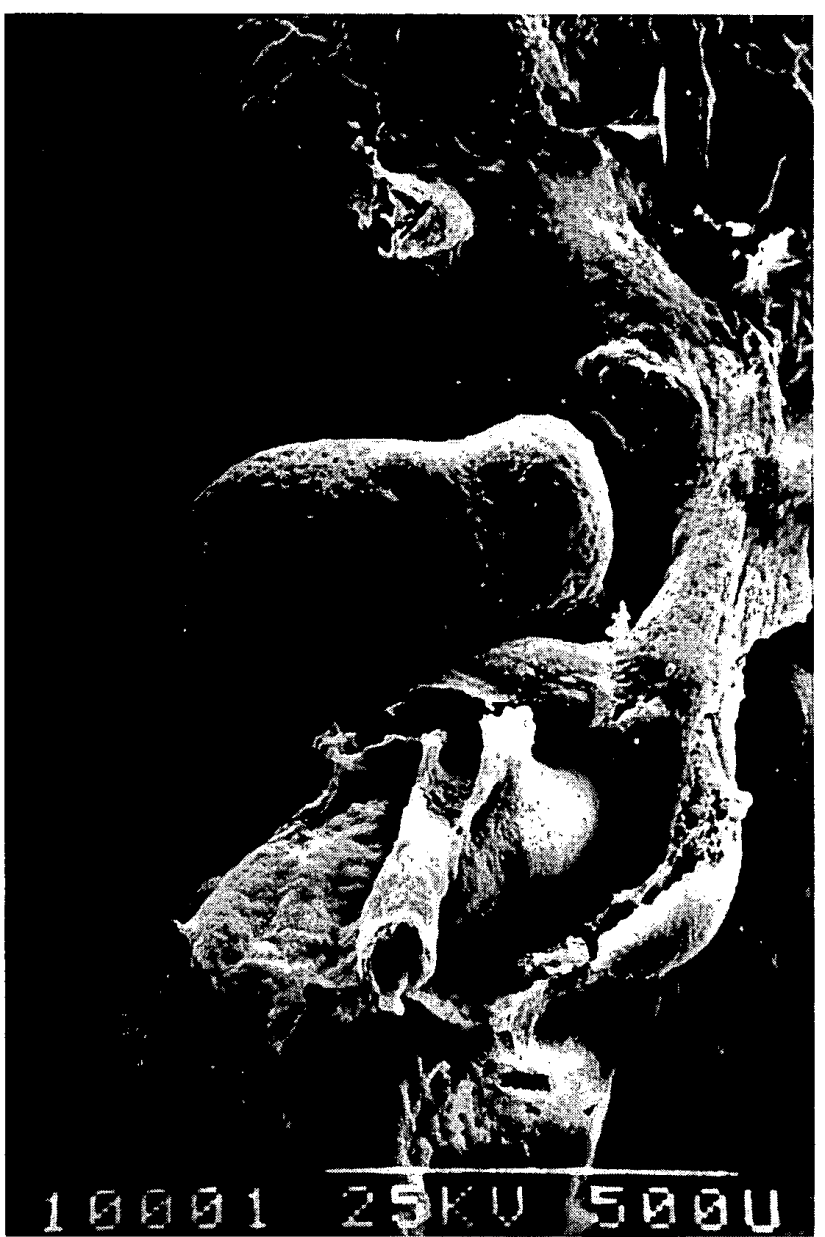

Fig.7. This SEM photograph reveals an abnormally positioned cardiac loop that is displaced to the right and rotated upwards.

straddling or overriding $\mathrm{AV}$ valves were observed. Although the left or right ventricle appeared hypoplastic, the outflow or inflow tract of the ventricles was only slightly elongated. Isolated dextrocardia became manifested in $82 \%(18 / 22)$ of the embryos.

Group III exhibited Double Outlet Right Ventricle (DORV) without pulmonary stenosis $(7 / 123=6 \%)$. Although dextrocardia was noted in $86 \%(6 / 7)$, only one case developed mesocardia.

In Group IV, a small subcristal VSD was noted with only a minor aortic arch anomaly $(10 / 123=8 \%)$. However, no abnormality of the cardiac apex was found in this group.

After application of the vibratory stimulus, SEM was performed to visualize the external morphology of the cardiac loop (Fig. 7). The distorted cardiac loop is clearly displaced to the right and rotated upwards. 


\section{DISCUSSION}

Data from this study clearly indicate that the application of vibration to the developing embryonic chick heart causes cardiovascular defects $(p<0.001)$. We observed several types of malformations, namely, straddling $\mathrm{AV}$ valves, variable sizes of VSD, and DORV. It is also noteworthy that the VSD incidence between treated and control embryos was statistically significant $(p<0.01)$. The most frequent and striking cardiac malformations in our experiments were positional anomalies, namely, isolated dextrocardia with straddling AV valves. Unlike earlier reports, we could not induce the various types of cardiac positional anomalies using vibratory mechanical interference, for two reasons. With regard to the spectrum of anomalies in earlier studies, irradiation was employed to induce an interruption of the early cardiac loop. It seems reasonable to assume that because we applied vibration to the embryo after the initial stages of cardiac loop formation, we could not produce more complicated positional anomalies. Secondly, we found that mechanical intervention does not elicit massive cellular changes or damage in the cardiac loop. In our experiments, the transformation from a straight to a distinct " $\mathrm{C}$ shaped" loop was only partially distorted by vibratory stimuli. Furthermore, with regard to the pathogenesis of intracardiac anomalies, the spectrum of morphogenetically straddling AV valves has been previously accounted for by an abnormal shift of the ventricular septum in the early embryonic heart? It is interesting to note that hypoplastic right and left ventricles with straddling AV valves have also been described. It is possible that one might induce various degrees of straddling AV valves by simply changing the frequency or strength of vibration applied to the embryo.

In our experiments, we also found several cases of mesocardia in the chick embryo. In fact, our embryonic chick studies were very similar to human autopsy findings?

Several other possibilities exist as to the pathogenesis of these vibration-induced malformations. One might suggest by deduction that they may be due to transient alterations of myocardial contractility or blood flow. However, we do not believe that this, per se, is a cause of these defects, for two reasons. One, the duration of the vibratory stimulus applied to the embryonic circulatory system was relatively brief in our experiments $(t=90$ seconds/egg). Although we may have momentarily disrupted blood flow patterns, this is unlikely to have been the direct cause of our induced malformations. Two, Manasek et al ${ }^{10}$ have demonstrated that early cardiac growth does not depend on hemodynamic factors. Thus, he has suggested that physiological development and cytodifferentiation continues despite alterations of normal function!1 Consequently, we may infer that vibration probably does not modify early myocardial development by altering cardiac function.

Other more plausible and logical explanations might be that vibration causes deforming forces upon the myofibrillar network, thereby allowing malformations to occur. A discussion of myocardial forces within the heart is presented by Nakamura et al. ${ }^{12}$ Recent work in their laboratory has supported the view that mechanical factors are important in determining myofibrillar cytoskeletal structure and arrangement. In addition, other workers have demonstrated that there may be abnormal alterations in heart cell shape and malalignment ${ }^{13}$ during morphogenesis that may ultimately lead to the production of cardiovascular anomalies. The crucial importance of regional changes in cell shape and alignment during normal cardiac development has been emphasized by Manasek et al $!^{14}$ Another hypothesis is related to a stochastic model that has been proposed by Kurnit et al ${ }^{14}$ whereby increased endocardial cushion cell adhesiveness in Trisomy 21 has resulted in a common AV canal. It is possible that vibration may increase cellular adhesiveness which would, in turn, lead to deficient endocardial cushion-interventricular septal fusion and result in a VSD. Additional studies concerning the precise histological alterations produced by vibration will be evaluated in our laboratory in the future.

\section{Acknowledgements}

This study was supported in part by: The Japanese Heart Foundation; Education Ministry of Japan /Grant \#442400138489); and a Prize from the Showa Medical Association.

\section{REFERENCES}

1. OKAMOTO N: Congenital anomalies of the heart. Embryologic, morphologic and experimental teratology. Igaku-Shoin, New York, 1980

2. DeHAAN RL: Morphogenesis of the vertebrate heart. In Organogenesis, ed by DeHAAN RL, URSPRUNG H, Holt, Rinehart, and Winston, New York, 1965

3. RYCHTER Z: Experimental morphology of the 
aortic arches and the heart loop in chick embryos. Adv Morph 2: 333, 1962

4. JAFFEE OC: Hemodynamic factors in the development of the chick embryo heart. Anat Rec 151: 69,1965

5. GESSNER IH: Spectrum of congenital heart anomalies produced in chick embryos by mechanical interference with cardiogenesis. Circ Res 18: 625,1966

6. GESSNER IH, VAN MIEROP LHS: Experimental production of cardiac defects: The spectrum of dextroposition of the aorta. Am $J$ Cardiol 25: 272,1970

7. HAMBURGER V, HAMILTON HL: A series of normal stages in the development of the chick embryo. J Morpho 88: 49, 1951

8. ANDO M, SATOMI G, TAKAO A: Atresia of tricuspid or mitral orifice: Anatomic spectrum and morphogenetic hypothesis. In Etiology and Morphogenesis of Congenital Heart Disease, ed by VAN PRAAGH, TAKAO A, Futura Publishing Co., Mt. Kisco, New York, 1980

9. LEV M, LIBERTHSON RR, GOLDEN JG, ECKNER FA, ARCILLA RA: The pathologic anatomy of mesocardia. Am J Cardiol 28: 428. 1971

10. MANASEK FJ, BURNSIDE M, VAN PRAAGH R: Heart looping and fibril formation. Circulation 44 (Suppl. II): II-1 16 (Abstract \#422), 1971

11. MANASEK FJ, MONROE RG: Early cardiac morphogenesis is independent of function. Dev Biol 27: 584, 1972

12. NAKAMURA A, KULIKOWSKI R, LACKTIS J, MANASEK FJ: Heart looping: A regulated response to deforming forces. In Etiology and Morphogenesis of Congenital Heart Disease, ed by VAN PRAAGH, TAKAO A, Futura Publishing Co., Mt. Kisco, New York, 1980

13. STALSBERG $\mathrm{H}$ : Mechanism of dextral looping in the embryonic heart. Am J Cardiol 25: 265, 1970

14. MANASEK FJ, BURNSIDE M, WATERMAN R: Myocardial cell shape change as a mechanism of embryonic heart looping. Dev Biol 29: 349, 1972

15. KURNIT DM, ALDRIDGE JF, MATSUOKA R, MATTHYSSE S: Increased adhesiveness of Trisomy 21 cells and atrioventricular canal malformations in Down Syndrome: A stochastic model. Am J Med Gen 20: 385, 1985 